\title{
Gypenoside attenuates renal ischemia/reperfusion injury in mice by inhibition of ERK signaling
}

\author{
QIFA YE, YI ZHU, SHAOJUN YE, HONG LIU, XINGGUO SHE, YING NIU and YINGZI MING

\begin{abstract}
Center of Transplant Medicine Engineering and Technology of Ministry of Health of The People's Republic of China,
\end{abstract} \\ The Third Xiangya Hospital, Central South University, Changsha, Hunan 410013, P.R. China
}

Received March 19, 2015; Accepted November 5, 2015

DOI: $10.3892 / \mathrm{etm} .2016 .3034$

\begin{abstract}
Gynostemma pentaphyllum is a traditional Chinese medicine reported to possess a wide range of health benefits. As the major component of G. pentaphyllum, gypenoside (GP) displays various anti-inflammatory and anti-oxidant properties. However, it is unclear whether GP can protect against ischemia/reperfusion (I/R)-induced renal injury, and the underlying molecular mechanisms associated with this process remain unknown. In the present study, a renal $\mathrm{I} / \mathrm{R}$ injury model in C57BL/6 mice was established. It was observed that, following I/R, serum concentrations of creatinine $(\mathrm{Cr})$ and blood urea nitrogen (BUN) were significantly increased $(\mathrm{P}<0.01)$, indicating renal injury. Pretreatment with GP (50 mg/kg) significantly inhibited I/R-induced upregulation of serum $\mathrm{Cr}$ and BUN $(\mathrm{P}<0.01)$. Furthermore, renal malondialdehyde levels were significantly reduced in the I/R+GP group, compared with the I/R group $(\mathrm{P}<0.01)$, whereas renal tissue superoxide dismutase activity was significantly higher in the I/R+GP group compared with the I/R group $(\mathrm{P}<0.01)$. Further investigation demonstrated that pretreatment with GP produced inhibitory effects on the I/R-induced production of pro-inflammatory cytokines, including interleukin (IL)-1 $\beta$, IL-6, tumor necrosis factor- $\alpha$ and interferon- $\gamma$ $(\mathrm{P}<0.01)$. In addition, heme oxygenase 1 (HO-1) expression levels were significantly increased in the I/R group compared with the control $(\mathrm{P}<0.01)$, indicating the presence of oxidative damage. However, the I/R-induced upregulation of HO-1 was significantly attenuated by pretreatment with GP $(\mathrm{P}<0.01)$, which also suppressed I/R-induced apoptosis by inhibiting pro-apoptotic Bax and upregulating anti-apoptotic Bcl-2 in renal cells $(\mathrm{P}<0.01)$. Finally, the activity of ERK signaling
\end{abstract}

Correspondence to: Professor Yingzi Ming, Center of Transplant Medicine Engineering and Technology of Ministry of Health of the People's Republic of China, The Third Xiangya Hospital of Central South University, 138 Tongzipo Road, Changsha, Hunan 410013, P.R. China

E-mail: doctormingyingzi@163.com

Key words: geniposide, renal injury, inflammation, apoptosis, ischemia/reperfusion was significantly increased in the I/R+GP group compared with the I/R group $(\mathrm{P}<0.05)$, which may be associated with the protective effect of GP against I/R-induced renal cell apoptosis. To conclude, the present results suggest that GP produces a protective effect against I/R-induced renal injury as a result of its anti-inflammatory and anti-apoptotic properties.

\section{Introduction}

Ischemia/reperfusion (I/R) can occur following renal surgery or transplantation, often resulting in acute kidney injury, chronic renal failure and kidney transplantation failure $(1,2)$. It has been demonstrated that inflammation and apoptosis serve crucial functions in I/R-induced renal injury (3), therefore the development of effective drugs to prevent inflammation and apoptosis in I/R-induced renal injury is required.

Gynostemma pentaphyllum is a traditional Chinese medicine that has previous been used for the treatment of renal diseases $(4,5)$. The major component of $G$. pentaphyllum is gypenoside (GP), which exhibits anti-inflammatory, antitumor and anti-oxidative properties (6-8). In particular, the protective effect of GP against I/R-induced injury has been reported. For example, Qi et al (9) reported that GP can protect neuronal DNA against the damage resulting from I/R-induced cerebral injury. Furthermore, it has been demonstrated in mice that GP exerts a therapeutic effect on chronic renal injury, fibrosis and fatty renal disease induced by alcohol consumption and a diet high in fat and cholesterol $(10,11)$. In addition, the authors of the present study previously observed that GP attenuated hepatic I/R injury in mice via anti-oxidative and anti-apoptotic bioactive characteristics (12). However, it remains unclear whether GP is able to exert a protective effect against I/R-induced renal injury, and the molecular mechanisms potentially underlying this process are as yet unknown.

Previous studies demonstrated that the inflammatory response was significantly upregulated following I/R (13). In addition, the inflammatory response to hypoxia has been shown to contribute to the resulting renal tissue injury (13). Inflammation-associated factors include complement proteins and chemokines, including tumor necrosis factor- $\alpha$ (TNF- $\alpha$ ), interferon (IFN) $-\gamma$, interleukin (IL)-1 $\beta$ and IL-6 (14). Inhibition of their production has been shown to attenuate 
I/R-induced tissue injury (15); in a previous study, catalpol protected mice against renal I/R injury by suppressing the phosphoinositide-3-kinase/Akt-endothelial nitric oxide synthase signaling pathway, as well as the expression of TNF- $\alpha$, IL-1 $\beta$, IL-6 and IL-10 (16). Furthermore, gypenoside was shown to exert suppressive effects on the expression of inflammatory molecules (8), and thus, may have an inhibitory role in I/R-induced renal injury.

In the present study, the protective effect of GP against I/R-induced renal injury was evaluated using a renal I/R injury model in C57BL/6 mice. In addition, the underlying molecular mechanisms involving inflammation and apoptosis in renal $\mathrm{I} / \mathrm{R}$ injury were investigated.

\section{Materials and methods}

Renal I/R injury model. A total of 30 male C57BL/6 mice (age, 12 weeks; weight, 20-25 g) were obtained from the Animal Center of Central South University (Changsha, China). The mice were housed at the Xiangya Medical Experimental Animal Center of the Central South University in a laminar flow, temperature-controlled, pathogen-free environment with a $12 \mathrm{~h}$ light/dark cycle and with ad libitum access to food and water. Mice were fasted for $24 \mathrm{~h}$ prior to the renal $\mathrm{I} / \mathrm{R}$ procedure, then an intraperitoneal injection of pentobarbital $(50 \mathrm{mg} / \mathrm{kg})$ was administered to anesthetize the animals. The animal experiment was approved by the Ethics Committee of The Third Xiangya Hospital of Central South University.

Administration of GP. GP was purchased from the China National Pharmaceutical Group Corporation (Beijing, China) and dissolved in saline in accordance with the manufacturer's instructions. Mice were divided into three groups, with each group containing 5 mice. In the sham (control) group, the mice underwent anesthesia with $4 \%$ chloral hydrate $(8 \mu \mathrm{l} / \mathrm{g}$; Dalian Meilun Biology Technology Co., Ltd., Dalian, China) and the renal I/R procedure, which included bilateral flank incisions and a right nephrectomy. In the GP-treated I/R (I/R+GP) group, mice were intravenously injected with $50 \mathrm{mg} / \mathrm{kg}$ GP using an infusion pump $1 \mathrm{~h}$ prior to the renal I/R procedure. In the saline-treated I/R group (I/R), mice were administered $50 \mathrm{mg} / \mathrm{kg}$ saline using an infusion pump $1 \mathrm{~h}$ prior to the renal I/R procedure.

Assessment of kidney function. Renal function was assessed by measuring serum creatinine $(\mathrm{Cr})$ and blood urea nitrogen (BUN) levels. These tests were performed by technicians at the Clinical Laboratory at The Third Xiangya Hospital, Central South University.

Assessment of renal malondialdehyde (MDA) expression levels. Homogenization buffer (Cayman Chemical Company, Ann Arbor, MI, USA), which contained $0.32 \mathrm{mmol} / 1$ sucrose, $20 \mathrm{mmol} / \mathrm{l} \mathrm{N}$-(2-hydroxyethyl)piperazine-N-(2-ethanesulfonic acid), $0.5 \mathrm{mmol} / 1$ ethylenediaminetetraacetic acid, $1 \mathrm{mmol} / \mathrm{l}$ 1,4-dithio-DL-threitol and $1 \mathrm{mmol} / 1$ phenylmethanesulfonyl fluoride, was added to renal tissue $(1 \mathrm{ml} / 0.1 \mathrm{~g})$. Following homogenization, renal tissue was centrifuged at $1,500 \mathrm{x} \mathrm{g}$ for $10 \mathrm{~min}$. According to Esterbauer and Cheeseman (17), MDA in tissue reacts with thiobarbituric acid in the sample, and thus, the changes in fluorescence as a result of this were detected at a wavelength of $535 \mathrm{~nm}$ in the present study. Recorded concentrations of MDA were divided by 1,000, and the results were expressed in $\mu \mathrm{mol} / \mathrm{g}$ wet tissue.

Assessment of renal tissue superoxide dismutase (SOD) activity. SOD activity was measured using a Superoxide Dismutase Activity Assay kit (BioVision, Inc., Milpitas, CA, USA), based on the inhibition of adenochrome production by SOD by adenochrome production during epinephrine auto-oxidation. Changes in fluorescence were detected at a wavelength of $480 \mathrm{~nm}$ using the $721 \mathrm{~S}$ Spectrophotometer (Shanghai Lengguang Industrial Co., Ltd., Shanghai, China).

Reverse transcription-quantitative polymerase chain reaction $(R T-q P C R)$ assay. Total RNA was extracted using TRIzol ${ }^{\circledR}$ reagent (Invitrogen; Thermo Fisher Scientific, Inc., Carlsbad, CA, USA). The RevertAid Reverse Transcription kit (Thermo Fisher Scientific, Inc.,) was used to convert RNA (1 $\mu \mathrm{g})$ into cDNA, according to the manufacturer's instructions. qPCR was then conducted using an Applied Biosystems 7500 thermocycler (Thermo Fisher Scientific, Inc.) and $0.33 \mu \mathrm{l}$ cDNA solution, $10 \mu \mathrm{l}$ SYBR Green PCR Master Mix (Thermo Fisher Scientific, Inc.), $2 \mu \mathrm{l}$ primers (Sangon Biotech Co., Ltd., Shanghai, China) and $7.67 \mu \mathrm{H}_{2} \mathrm{O}$, to obtain a final reaction volume of $20 \mu \mathrm{l}$. The primers used for the qPCR were as follows: TNF- $\alpha$ forward, 5'-CAGGCGGTGCCTATGTCTC-3' and reverse, 5'-CGATCACCCCGAAGTTCAGTAG-3'; IFN- $\gamma$ forward, 5'-GCCACGGCACAGTCATTGA-3' and reverse, 5'-TGCTGATGGCCTGATTGTCTT-3'; IL-1 $\beta$ forward, 5'-GAAATGCCACCTTTTGACAGTG-3' and reverse, 5'-TGG ATGCTCTCATCAGGACAG-3'; IL-6 forward, 5'-CTGCAA GAGACTTCCATCCAG-3' and reverse, 5'-AGTGGTATA GACAGGTCTGTTGG-3'; and GAPDH forward, 5'-TGACCT CAACTACATGGTCTACA-3' and reverse, 5'-CTTCCCATT CTCGGCCTTG-3'. The cycling conditions were as follows: $95^{\circ} \mathrm{C}$ for $10 \mathrm{~min}$, followed by 40 cycles of denaturation at $95^{\circ} \mathrm{C}$ for $15 \mathrm{sec}$ and annealing/elongation at $60^{\circ} \mathrm{C}$ for $60 \mathrm{sec}$. GAPDH was used as an internal reference. The relative expression levels were analyzed by the Applied Biosystems 7500 Fast Real-Time PCR System (Thermo Fisher Scientific, Inc.), using the $2^{-\Delta \Delta \mathrm{Cq}}$ method (18).

Measurement of serum proinflammatory cytokine levels. Enzyme-linked immunosorbent assay (ELISA) kits (Sigma-Aldrich, St. Louis, MO, USA) were used to determine the serum concentration levels of various key proinflammatory cytokines, including TNF- $\alpha$ (RAB0477), IFN- $\gamma$ (RAB0224), IL-1 $\beta$ (RAB0274) and IL-6 (RAB0308), in accordance with the manufacturer's instructions.

Western blot analysis. The NE-PER ${ }^{\circledR}$ Nuclear and Cytoplasmic Extraction reagents (Pierce Biotechnology, Inc., Rockford, IL, USA) were used to extract cytosolic proteins from mice renal tissue, in accordance with the manufacturer's protocol. Protein assay reagents (Beyotime Institute of Biotechnology, Haimen, China) were used to determine protein concentration, after which $20 \mu \mathrm{g}$ protein was separated by $10 \%$ SDS-PAGE (initially $80 \mathrm{~V}$ and then $150 \mathrm{~V}$; Beyotime Institute of Biotechnology), transferred to nitrocellulose membranes (Thermo Fisher Scientific, Inc.) and maintained at room temperature for $1 \mathrm{~h}$ 
A

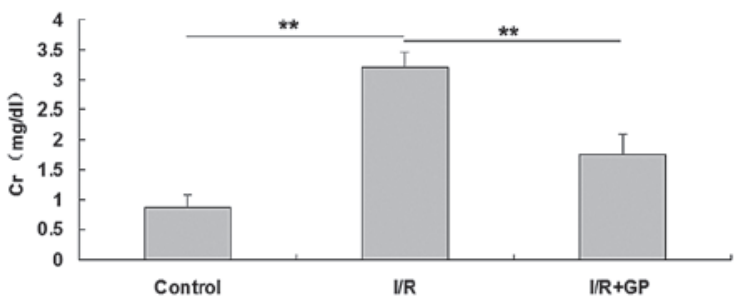

$\mathbf{B}$

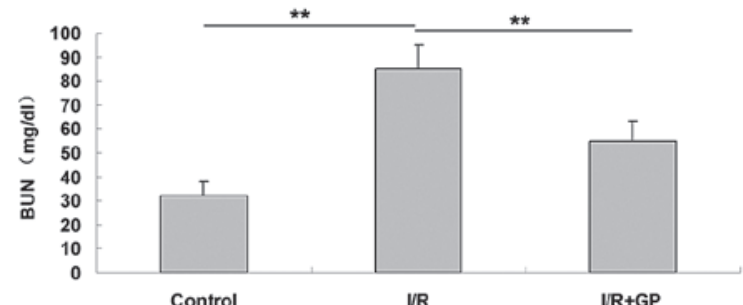

C
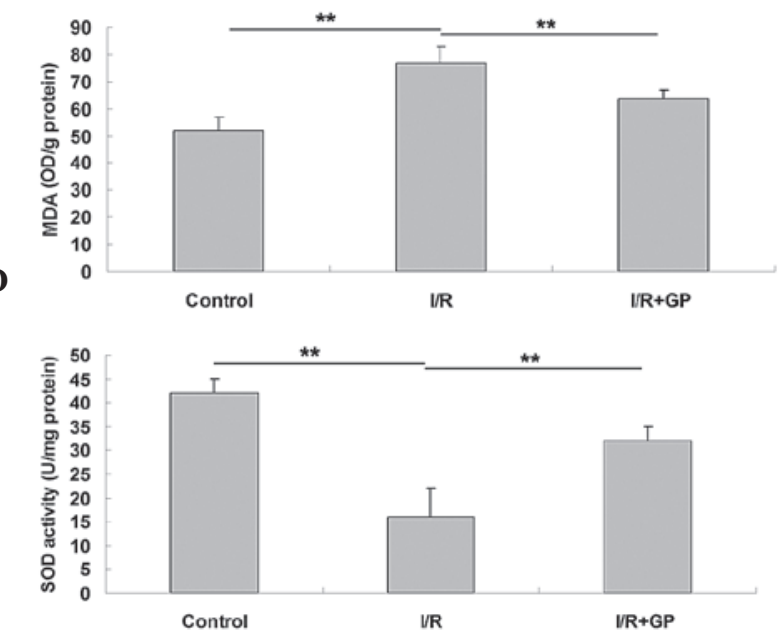

Figure 1. Renal tissue serum concentration of (A) CR, (B) BUN, (C) MDA and (D) SOD. Data are presented as the mean \pm standard error. ${ }^{* *} \mathrm{P}<0.01$, comparisons shown by brackets. I/R, saline-treated ischemia/reperfusion group; I/R+GP, geniposide-treated ischemia/reperfusion group; $\mathrm{CR}$, creatinine; BUN, blood urea nitrogen; MDA, malondialdehyde; SOD, superoxide dismutase.

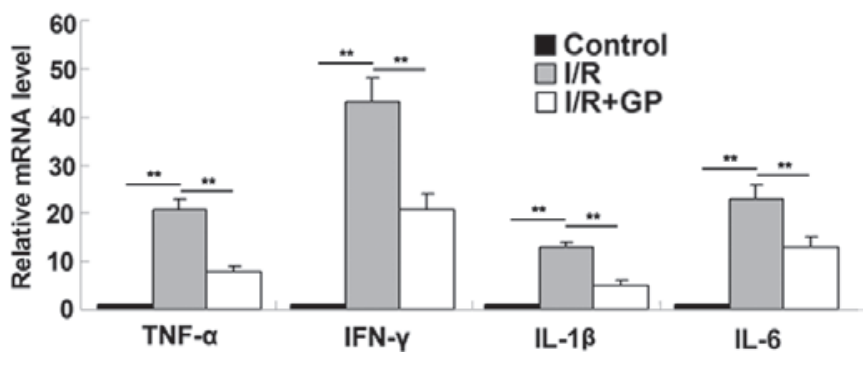

Figure 2. Renal tissue mRNA expression levels of key proinflammatory cytokines. Data are presented as the mean \pm standard error. ${ }^{* *} \mathrm{P}<0.01$, comparisons as shown by brackets. TNF- $\alpha$, tumor necrosis factor- $\alpha$; IFN- $\gamma$, interferon- $\gamma$; IL-1 $\beta$, interleukin-1 $\beta$; IL-6, interleukin-6; I/R, saline-treated ischemia/reperfusion group; I/R+GP, geniposide-treated ischemia/reperfusion group.

in a buffer solution containing $5 \%$ dried skim milk. The membrane was then incubated at room temperature for $3 \mathrm{~h}$ with the following antibodies from Abcam (Cambridge, MA, USA): Polyclonal rabbit anti-human p-ERK (1:100; ab65142), ERK
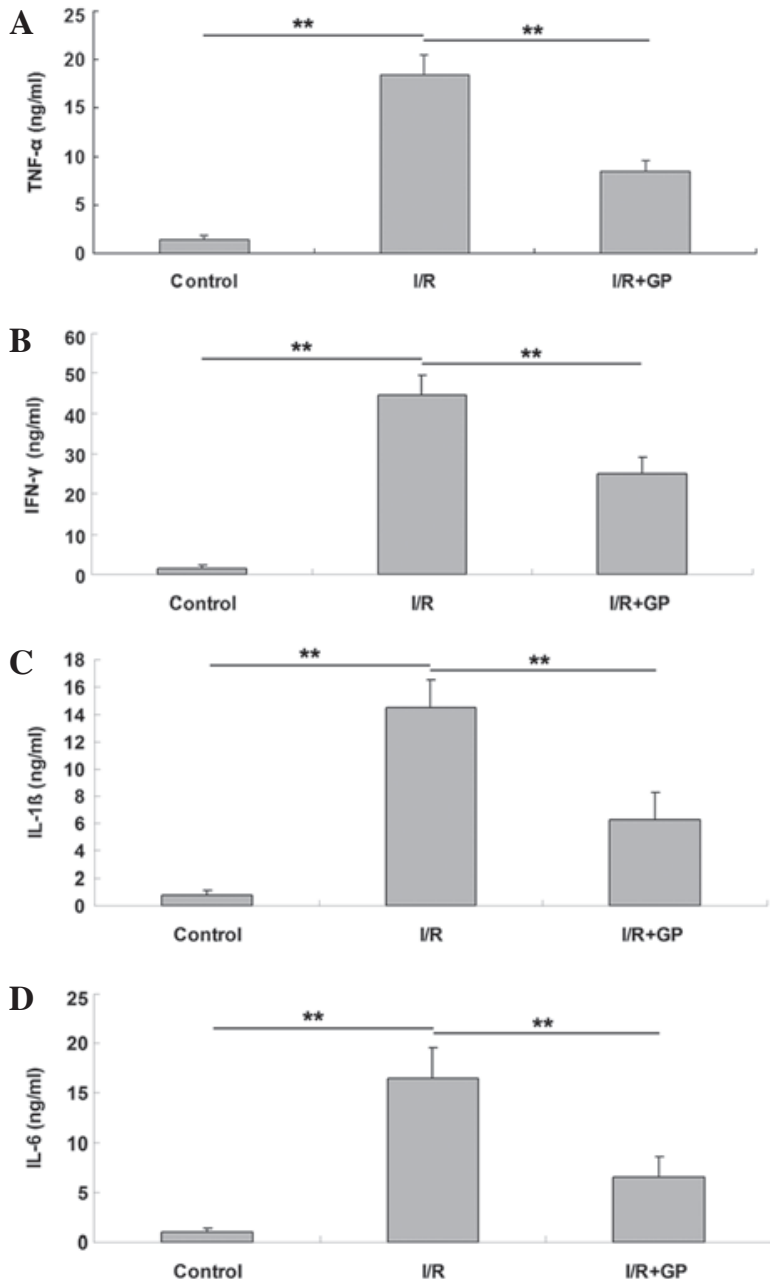

Figure 3. Renal tissue expression levels of proinflammatory cytokines, including (A) TNF- $\alpha$, (B) IFN- $\gamma$, (C) IL-1 $\beta$ and (D) IL- 6 . Data are presented as the mean \pm standard error. ${ }^{* *} \mathrm{P}<0.01$, comparisons as shown by brackets. TNF- $\alpha$, tumor necrosis factor- $\alpha$; IFN- $\gamma$, interferon- $\gamma$; IL- $1 \beta$, interleukin- $1 \beta$; IL-6, interleukin-6; I/R, saline-treated ischemia/reperfusion group; I/R+GP, geniposide-treated ischemia/reperfusion group.

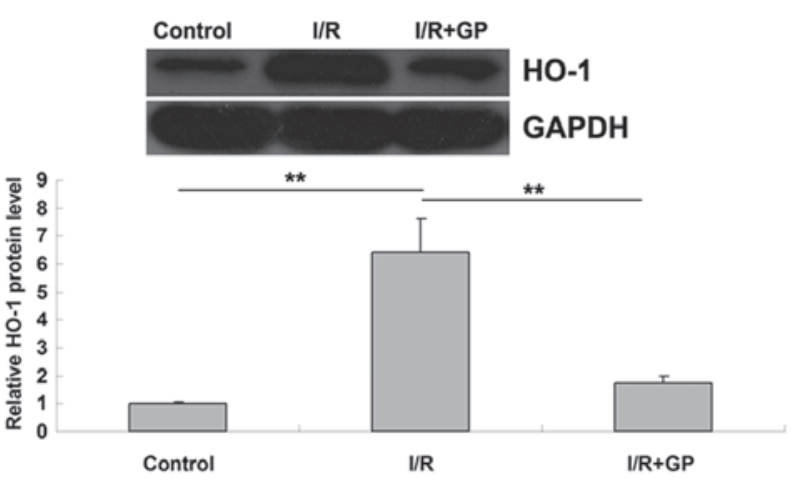

Figure 4. Protein expression levels of HO-1 in renal tissue. Data are presented as the mean \pm standard error. ${ }^{* *} \mathrm{P}<0.01$, comparisons shown by brackets. GAPDH was used as an internal control. HO-1, heme oxygenase-1; I/R, saline-treated ischemia/reperfusion group; I/R+GP, geniposide-treated ischemia/reperfusion group.

(1:200; ab32537), heme oxygenase-1 (HO-1; 1:200; ab13248), Bcl-2 (1:100; ab117115), Bax (1:50; ab79459) and GAPDH (1:50; ab181602). Subsequently, the samples were incubated for $1 \mathrm{~h}$ with horseradish peroxidase-conjugated goat anti-rabbit 

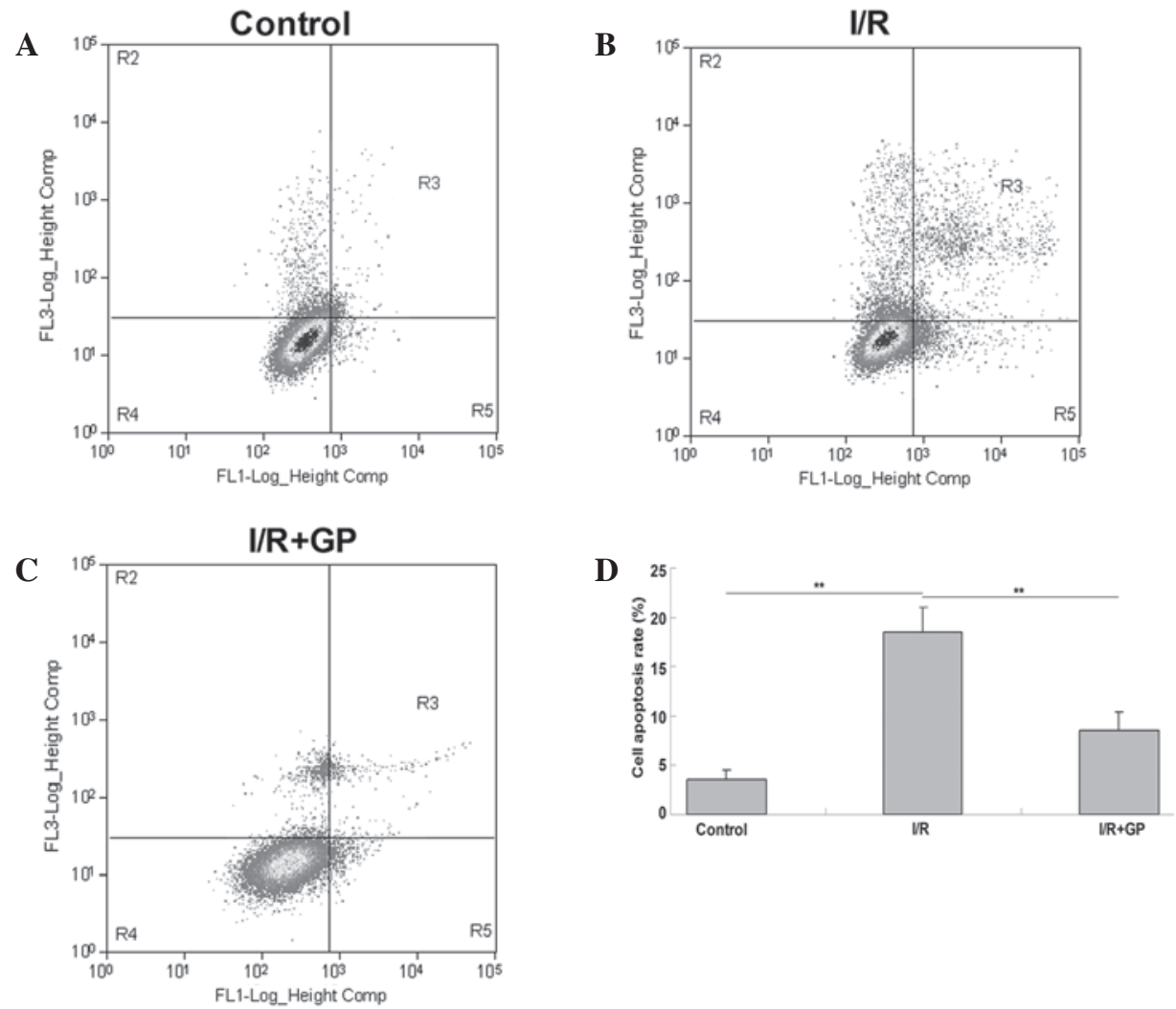

Figure 5. An apoptosis assay performed in renal tissues in the (A) control, (B) I/R and (C) I/R+GP; (D) quantification of apoptosis in each group. Data are presented as the mean \pm standard error. ${ }^{* * *} \mathrm{P}<0.01$, comparisons shown by brackets. I/R, saline-treated ischemia/reperfusion; I/R+GP, geniposide-treated ischemia/reperfusion group.

IgG (1:20,000; ab6721). The signals on the membranes were detected using enhanced chemiluminescence reagent (Pierce Biotechnology, Inc.) and densitometry was conducted using Image-Pro Plus software, version 6.0 (Media Cybernetics, Inc., Rockville, MD, USA).

Apoptosis analysis. Cell apoptosis was analyzed using the Annexin V-FITC Apoptosis Detection kit (BD Biosciences, Franklin Lakes, NJ, USA), according to the manufacturer's protocol. Briefly, at $24 \mathrm{~h}$ post-transfection, the cells were harvested and washed twice with cold PBS. Subsequently, $10^{6}$ cells were resuspended in $200 \mu \mathrm{l}$ binding buffer added to $10 \mu \mathrm{l}$ Annexin-V-FITC and $5 \mu \mathrm{l}$ PI-PE, followed by incubation in the dark for $30 \mathrm{~min}$. Finally, $300 \mu \mathrm{l}$ binding buffer was added, followed by the flow cytometry assay.

Statistical analysis. All data are presented as the mean \pm standard error, and analyzed using one-way analysis of variance. Statistical analyses were conducted using SPSS software, version 16.0 (SPSS, Inc., Chicago, IL, USA). P<0.05 was considered to indicate a statistically significant difference.

\section{Results}

GP exerted a protective effect on I/R-induced renal injury in mice. Serum concentrations of $\mathrm{Cr}$ and BUN were measured following I/R-induced renal injury, and it was observed that mice in the $\mathrm{I} / \mathrm{R}$ group presented significantly higher serum levels of $\mathrm{Cr}$ and BUN compared with the control group $(\mathrm{P}<0.01$;
Fig. 1A and B), indicating the occurrence of I/R-induced renal injury. In the I/R+GP group, serum concentration levels of $\mathrm{Cr}$ and BUN were significantly reduced compared with the I/R group $(\mathrm{P}<0.01$; Fig. $1 \mathrm{~A}$ and $\mathrm{B})$, suggesting that pretreatment with GP has a protective effect against I/R-induced renal injury. However, the MDA concentration in the renal tissue at $6 \mathrm{~h}$ after reperfusion was significantly increased in the I/R group compared with the control and I/R+GP groups (Fig. 1C). In addition, SOD activity was higher in the I/R+GP group compared with the I/R group (Fig. 1D).

Pretreatment with GP attenuated I/R-induced inflammatory responses in the kidney. The inflammatory responses resulting from $\mathrm{I} / \mathrm{R}$ in kidney tissue were examined in mice. It was observed that the mRNA expression levels of the inflammatory cytokines TNF- $\alpha$, IFN- $\gamma$, IL- $1 \beta$ and IL- 6 were higher in renal tissue following I/R compared with the control and I/R+GP groups (Fig. 2). To further confirm these results, an ELISA was performed, which demonstrated that the secretion levels of TNF- $\alpha$, IFN- $\gamma$, IL- $1 \beta$ and IL- 6 were significantly increased in renal tissue following $\mathrm{I} / \mathrm{R}$, but reduced in the GP treatment group $(\mathrm{P}<0.01$; Fig. 3$)$.

Pretreatment with GP attenuated I/R-induced oxidative damage in the kidney. Heme oxygenase-1 (HO-1) is crucial in the defense against oxidative damage, and the expression of HO-1 can be induced by I/R (19). In the present study, HO-1 protein expression was detected using western blot analysis. The results indicated that HO-1 expression was 
A
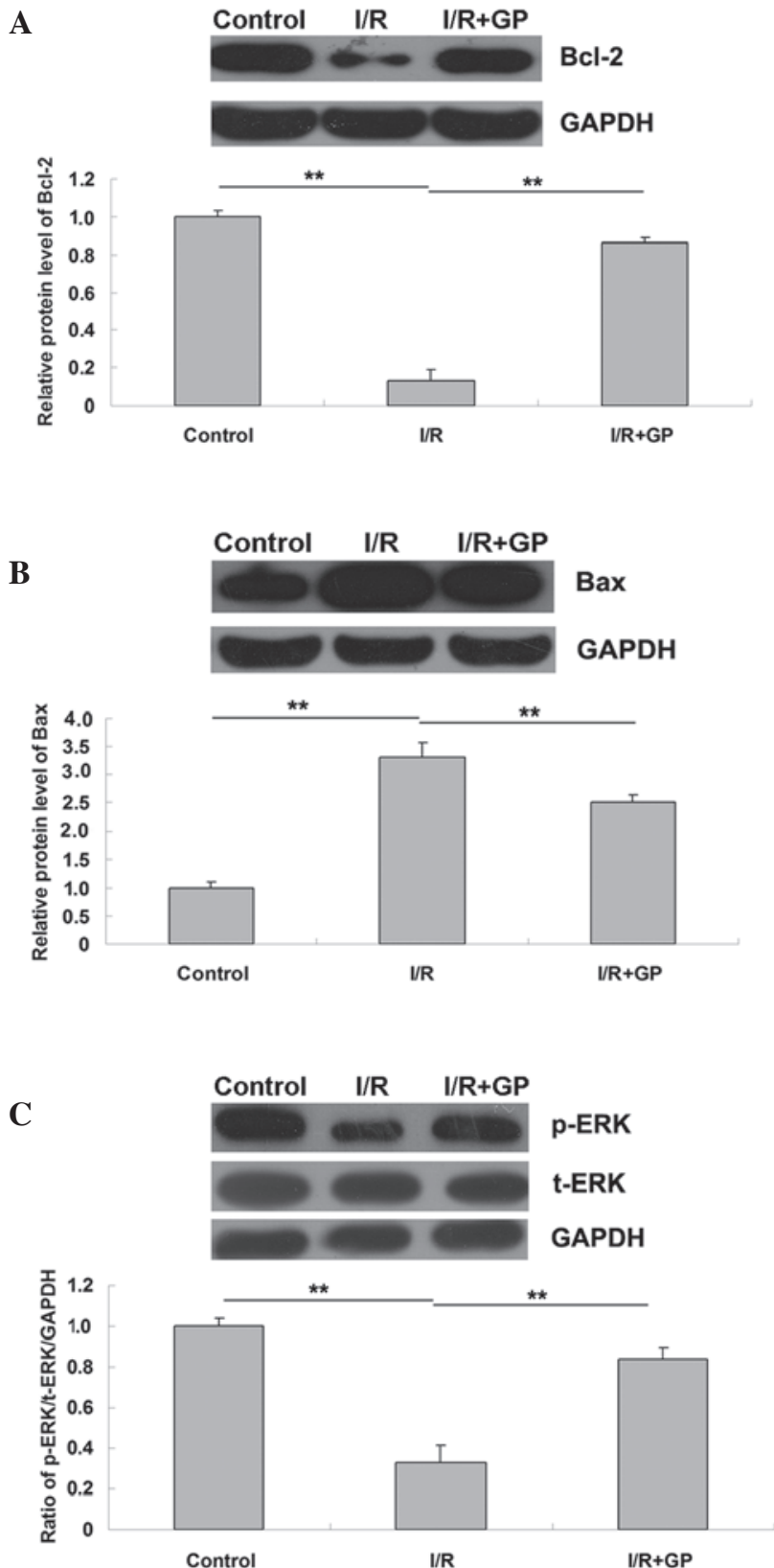

Figure 6. Renal tissue protein expression levels of (A) Bcl-2, (B) Bax and (C) phospho-ERK and total-ERK. I/R, saline-treated ischemia/reperfusion group; I/R+GP, geniposide-treated ischemia/reperfusion group. Data are presented as the mean \pm standard error. ${ }^{* *} \mathrm{P}<0.01$, comparisons shown by brackets.

significantly increased in the $\mathrm{I} / \mathrm{R}$ group compared with the control group $(\mathrm{P}<0.01$; Fig. 4$)$, suggesting that oxidative damage had occurred. However, this I/R-induced upregulation of HO-1 was attenuated by pretreatment with GP; the IR+GP group exhibited significantly reduced levels of HO-1 protein, compared with the IR group $(\mathrm{P}<0.01 ;$ Fig. 4). It may therefore be suggested that pretreatment with GP attenuates I/R-induced oxidative damage in mice kidney tissue.

Pretreatment with GP attenuated I/R-induced apoptosis of renal cells. The effect of GP on I/R-induced renal cell injury was investigated using an apoptosis assay. As presented in Fig. 5, the level of apoptosis in kidney tissue following I/R was significantly increased compared with the control group
$(\mathrm{P}<0.01)$; however, pretreatment with GP significantly attenuated I/R-induced renal cell apoptosis ( $\mathrm{P}<0.01$ vs. I/R group).

Molecular mechanisms of GP. The expression levels of the apoptosis-associated $\mathrm{Bcl}-2$ and Bax proteins were examined. As demonstrated in Fig. 6A, the protein expression levels of anti-apoptotic Bcl-2 were significantly reduced in the $\mathrm{I} / \mathrm{R}$ group compared with the control group $(\mathrm{P}<0.01)$. However, the expression level was higher in the $\mathrm{I} / \mathrm{R}+\mathrm{GP}$ group compared with the $\mathrm{I} / \mathrm{R}$ group $(\mathrm{P}<0.01)$. In addition, it was observed that the protein level of pro-apoptotic Bax was increased in the I/R group compared with the control group, and that Bax protein expression was attenuated by pretreatment with GP $(\mathrm{P}<0.01$; Fig. 6B). This suggests that the protective effect of GP against I/R-induced renal injury may be attributed to the inhibition of cell apoptosis in the kidney.

$G P$ suppresses I/R-induced downregulation of ERK signaling in renal tissues. As presented in Fig. $6 \mathrm{C}$, the expression level of phosphorylated ERK (p-ERK) in the I/R group was significantly reduced compared with the control group, and p-ERK was significantly attenuated following pretreatment with GP in the $\mathrm{I} / \mathrm{R}+\mathrm{GP}$ group $(\mathrm{P}<0.01)$. This suggests that the administration of GP suppressed I/R-induced the downregulation of ERK signaling in renal tissue, which may be associated with the protective effect of GP against I/R-induced renal cell apoptosis.

\section{Discussion}

It has previously been demonstrated that GP possesses various bioactive characteristics including anti-oxidative, anti-inflammatory, anti-apoptotic and antitumor properties $(9,11,20)$. In the present study, kidney tissue that had been pretreated with GP exhibited significantly inhibited I/R-induced upregulation of serum $\mathrm{Cr}$ and BUN protein expression. In addition, the I/R+GP group demonstrated significantly lower expression levels of MDA and higher SOD activity compared with the I/R group. Furthermore, pretreatment with GP inhibited the I/R-induced production of pro-inflammatory cytokines, oxidative damage and apoptosis. Investigation of the molecular mechanisms involved suggested that pretreatment with GP inhibits the I/R-induced activation of ERK signaling.

Inflammation has been recognized as a key mechanism underlying the pathogenesis of renal I/R injury (21). In response to $\mathrm{I} / \mathrm{R}$, neutrophils, lymphocytes and macrophages infiltrate the damaged tissue, which can lead to microcirculatory failure as a result of the reduction in renal blood flow $(22,23)$. Other proinflammatory factors, including TNF- $\alpha$, IFN- $\gamma$, IL- $1 \beta$ and IL-6 further promote the inflammatory response in renal tissue (24).

GP has been demonstrated to produce a suppressive effect on the I/R-induced inflammatory response. Quan et al (8) observed that treatment with GP inhibited the expression of inflammatory molecules, including intercellular adhesion molecule 1, monocyte chemotactic protein-1 and nuclear factor- $\mathrm{\kappa B}$, in atherosclerotic rats that were induced by a high-fat diet (8). Another previous study demonstrated that treatment with GP protects against I/R-induced hepatic injury by inhibiting neutrophil infiltration (12). In the present study, it was observed that the secretion of TNF- $\alpha$, IFN- $\gamma$, IL- $1 \beta$ and IL- 6 was significantly 
reduced in the $\mathrm{I} / \mathrm{R}+\mathrm{GP}$ group compared with the I/R group. Based on current knowledge, it may be hypothesized that the protective effect of GP against I/R-induced renal injury is partly caused by the suppression of inflammatory responses.

Cell death is frequently controlled by the Bcl-2 protein family, which contains pro-apoptotic and anti-apoptotic proteins (25). The Bcl-2 family serves a number of crucial functions in the control of cell survival during developmental and pathophysiological processes, including ischemic injury, neuronal $\mathrm{Ca}^{2+}$ homeostasis, trophic factor withdrawal, oxidative stress, excitotoxicity and energy stress (26). As an anti-apoptotic protein, Bcl-2 is able to inhibit lipid peroxide formation, $\mathrm{Ca}^{2+}$ release from endoplasmic reticulum and free radical production (27). Bax, another member of the Bcl-2 family, is an endogenous antagonist of Bcl-2 that inhibits the protein by directly binding via an associated protein homologue, thereby promoting cell apoptosis (28). However, Bcl-2 can reversely suppress the pro-apoptotic effect of Bax by binding to Bcl-xL and forming a heterodimer (29). The expression of Bcl-2 and Bax are carefully maintained and balanced in a healthy physiological state. However, the current study observed that $\mathrm{I} / \mathrm{R}$ activates renal cell apoptosis by increasing the protein expression levels of Bax and reducing the protein levels of Bcl-2. In addition, the present results demonstrated that $\mathrm{Bcl}-2$ can be attenuated by pretreating renal tissue with GP, suggesting that the protective effect of GP on I/R-induced renal cell apoptosis occurs via a Bcl-2-dependent mechanism.

Mitogen-activated protein kinases (MAPKs) have been demonstrated to be key factors in I/R-induced tissue damage. MAPK compounds, including ERK, stress-activated protein kinases/c-Jun $\mathrm{NH}_{2}$-terminal kinases and p38 MAPKs have been implicated in inflammatory signaling mechanisms in $\mathrm{I} / \mathrm{R}$ injury $(30,31)$. Additionally, ERK signaling serves a crucial function in I/R-induced cell apoptosis $(32,33)$. Ban et al (34) reported that the inhibition of ERK signaling worsened intestinal I/R injury. In addition, Yang et al (35) observed that Apelin-13 protects the brain against I/R injury by activating ERK signaling pathways. Furthermore, GP has been demonstrated to upregulate ERK signaling and inhibit JNK signaling in local cerebral I/R injury rats (36). In the present study, it was demonstrated that GP significantly suppresses the downregulation of p-ERK protein expression levels induced by I/R, indicating that GP activates ERK signaling. It may therefore be suggested that, in mice, the protective effect of GP on renal cell apoptosis occurs as a result of the upregulation of ERK signaling in I/R-induced kidney injury.

In conclusion, the current study demonstrates that the administration of GP exerts protective effects against I/R-induced renal injury in mice by attenuating inflammatory responses and suppressing apoptosis. As a result, it can be suggested that GP may be used in the treatment of renal I/R injury.

\section{Acknowledgements}

The current study was supported by the Scientific and Technological Brainstorm Project of Wuhan City (grant no. 201161038344-01), the Natural Science Fund of Hubei Province (grant no. 2012FFA044) and the Public Service Platform Construction Projects of Wuhan Technology Bureau (grant no. 2013060705010326).

\section{References}

1. Zhou M, Tang W,Fu Y, Xu X, Wang Z, Lu Y,Liu F, Yang X, Wei X, Zhang, Y et al: Progranulin protects against renal ischemia/reperfusion injury in mice. Kidney Int 87: 918-929, 2015.

2. Menke J, Sollinger D, Schamberger B, Heemann U and Lutz J: The effect of ischemia/reperfusion on the kidney graft. Curr Opin Organ Transplant 19: 395-400, 2014.

3. Kaczorowski DJ, Tsung A and Billiar TR: Innate immune mechanisms in ischemia/reperfusion. Front Biosci (Elite Ed) 1: 91-98, 2009.

4. Quan Y, Yang Y, Wang H, Shu B, Gong QH and Qian M: Gypenosides attenuate cholesterol-induced DNA damage by inhibiting the production of reactive oxygen species in human umbilical vein endothelial cells. Mol Med Rep 11: 2845-2851, 2015.

5. Deng $Q$ and Yang $X$ : Protective effects of Gynostemma pentaphyllum polysaccharides on PC12 cells impaired by MPP(+). Int J Biol Macromol 69: 171-175, 2014.

6. Li K, Du Y, Fan Q, Tang CY and He JF: Gypenosides might have neuroprotective and immunomodulatory effects on optic neuritis. Med Hypotheses 82: 636-638, 2014.

7. Yan H, Wang X, Niu J, Wang Y, Wang P and Liu Q: Anti-cancer effect and the underlying mechanisms of gypenosides on human colorectal cancer SW-480 cells. PLoS One 9: e95609, 2014.

8. Quan Y and Qian MZ: Effect and mechanism of gypenoside on the inflammatory molecular expression in high-fat induced atherosclerosis rats. Zhong Guo Zhong Xi Yi Jie He Za Zhi 30: 403-406, 2010 (In Chinese).

9. Qi G, Zhang L, Xie WL, Chen XY and Li JS: Protective effect of gypenosides on DNA and RNA of rat neurons in cerebral ischemia-reperfusion injury. Acta Pharmacol Sin 21: 1193-1196, 2000.

10. Zhang Y, Zhang JE, Xiao HQ, Wu PY and Bai SJ: Gypenosides inhibit renal fibrosis by regulating expression of related genes in rats with unilateral ureteral obstruction. J Nephrol 24: 112-118, 2011.

11. Qin R, Zhang J, Li C, Zhang X, Xiong A, Huang F, Yin Z, Li K, Qin W, Chen M, et al: Protective effects of gypenosides against fatty liver disease induced by high fat and cholesterol diet and alcohol in rats. Arch Pharm Res 35: 1241-1250, 2012.

12. Zhao J, Ming Y, Wan Q, Ye S, Xie S, Zhu Y, Wang Y, Zhong Z, Li L and Ye Q: Gypenoside attenuates hepatic ischemia/reperfusion injury in mice via anti-oxidative and anti-apoptotic bioactivities. Exp Ther Med 7: 1388-1392, 2014.

13. Daemen MA, de Vries B and Buurman WA: Apoptosis and inflammation in renal reperfusion injury. Transplantation 73: 1693-1700, 2002

14. Cao XZ, Ma H, Wang JK, Liu F, Wu BY, Tian AY, Wang LL and Tan WF: Postoperative cognitive deficits and neuroinflammation in the hippocampus triggered by surgical trauma are exacerbated in aged rats. Prog Neuropsychopharmacol Biol Psychiatry 34: 1426-1432, 2010

15. Facio FN Jr, Sena AA, Araújo LP, Mendes GE, Castro I, Luz MA, Yu L, Oliani SM and Burdmann EA: Annexin 1 mimetic peptide protects against renal ischemia/reperfusion injury in rats. J Mol Med (Berl) 89: 51-63, 2011.

16. Zhu J, Chen X, Wang H and Yan Q: Catalpol protects mice against renal ischemia/reperfusion injury via suppressing PI3K/Akt-eNOS signaling and inflammation. Int J Clin Exp Med 8: 2038-2044, 2015.

17. Esterbauer $\mathrm{H}$ and Cheeseman KH: Determination of aldehydic lipid peroxidation products: Malonaldehyde and 4-hydroxynonenal. Methods Enzymol 186: 407-421, 1990.

18. Livak KJ and Schmittgen TD: Analysis of relative gene expression data using real-time quantitative PCR and the 2(-Delta Delta C(T)) Method. Methods 25: 402-408, 2001

19. Sass G, Barikbin R and Tiegs G: The multiple functions of heme oxygenase-1 in the liver. Z Gastroenterol 50: 34-40, 2012.

20. Chen JC, Tsai CC, Chen LD, Chen HH and Wang WC: Therapeutic effect of gypenoside on chronic liver injury and fibrosis induced by CCl4 in rats. Am J Chin Med 28: 175-185, 2000.

21. El Morsy EM, Ahmed MA and Ahmed AA: Attenuation of renal ischemia/reperfusion injury by açaí extract preconditioning in a rat model. Life Sci 123: 35-42, 2014.

22. Ysebaert DK, De Greef KE, Vercauteren SR, Ghielli M, Verpooten GA, Eyskens EJ and De Broe ME: Identification and kinetics of leukocytes after severe ischaemia/reperfusion renal injury. Nephrol Dial Transplant 15: 1562-1574, 2000. 
23. Contaldo C, Meier C, Elsherbiny A, Harder Y, Trentz O, Menger MD and Wanner GA: Human recombinant erythropoietin protects the striated muscle microcirculation of the dorsal skinfold from postischemic injury in mice. Am J Physiol Heart Circ Physiol 293: H274-H283, 2007.

24. Domański L, Pawlik A, Safranow K, Rozański J, Myślak M, Sulikowski T, Romanowski M, Ostrowski M, Wiśniewska M, Domański $\mathrm{M}$, et al: Changes in cytokine concentrations in graft renal vein during reperfusion in patients with and without delayed graft function. Ann Acad Med Stetin 54: 49-52, 2008.

25. Akl H, Vervloessem T, Kiviluoto S, Bittremieux M, Parys JB De Smedt $\mathrm{H}$ and Bultynck $\mathrm{G}$ : A dual role for the anti-apoptotic Bcl-2 protein in cancer: Mitochondria versus endoplasmic reticulum. Biochim Biophys Acta 1843: 2240-2252, 2014.

26. Anilkumar U and Prehn JH: Anti-apoptotic BCL-2 family proteins in acute neural injury. Front Cell Neurosci 8: 281, 2014.

27. Saitoh Y, Ouchida R, Kayasuga A and Miwa N: Anti-apoptotic defense of bcl-2 gene against hydroperoxide-induced cytotoxicity together with suppressed lipid peroxidation, enhanced ascorbate uptake, and upregulated Bcl-2 protein. J Cell Biochem 89: 321-334, 2003

28. Renault TT and Chipuk JE: Death upon a kiss: Mitochondrial outer membrane composition and organelle communication govern sensitivity to BAK/BAX-dependent apoptosis. Chem Biol 21: 114-123, 2014.

29. Renault TT, Teijido O, Antonsson B, Dejean LM and Manon S: Regulation of Bax mitochondrial localization by Bcl-2 and $\mathrm{Bcl}-\mathrm{x}(\mathrm{L})$ : Keep your friends close but your enemies closer. Int J Biochem Cell Biol 45: 64-67, 2013.
30. Jiang M, Li J, Peng Q, Liu Y, Liu W, Luo C, Peng J, Li J, Yung KK and Mo Z: Neuroprotective effects of bilobalide on cerebral ischemia and reperfusion injury are associated with inhibition of pro-inflammatory mediator production and down-regulation of JNK1/2 and p38 MAPK activation. J Neuroinflammation 11: 167, 2014.

31. Jun JH, Jun NH, Shim JK, Shin EJ and Kwak YL: Erythropoietin protects myocardium against ischemia-reperfusion injury under moderate hyperglycemia. Eur J Pharmacol 745: 1-9, 2014.

32. Ottani A, Galantucci M, Ardimento E, Neri L, Canalini F, Calevro A, Zaffe D, Novellino E, Grieco P, Giuliani D and Guarini S: Modulation of the JAK/ERK/STAT signaling in melanocortin-induced inhibition of local and systemic responses to myocardial ischemia/reperfusion. Pharmacol Res 72: 1-8, 2013.

33. Wu X, Xu T, Li D, Zhu S, Chen Q, Hu W, Pan D, Zhu H and Sun H: ERK/PP1a/PLB/SERCA2a and JNK pathways are involved in luteolin-mediated protection of rat hearts and cardiomyocytes following ischemia/reperfusion. PLoS One 8: e82957, 2013

34. Ban K, Peng Z and Kozar RA: Inhibition of ERK1/2 worsens intestinal ischemia/reperfusion injury. PLoS One 8: e76790, 2013.

35. Yang Y, Zhang X, Cui H, Zhang C, Zhu C and Li L: Apelin-13 protects the brain against ischemia/reperfusion injury through activating PI3K/Akt and ERK1/2 signaling pathways. Neurosci Lett 568: 44-49, 2014.

36. Wang QY, Liu F, Wu FJ and Li JL: Effects of ginsenoside Rg1 on the expressions of $\mathrm{p}$-eRK $1 / 2$ and $\mathrm{p}-\mathrm{JNK}$ in local cerebral ischemia/reperfusion injury rats. Zhong Guo Zhong Xi Yi Jie He Za Zhi 33: 229-234, 2013 (In Chinese). 